\title{
Repeatability and Interchangeability of
}

\section{Topometric, Anterior Chamber and Corneal Wavefront Data Between Two Scheimpflug Camera Devices}

This article was published in the following Dove Press journal: Clinical Ophthalmology

\author{
Hesham Mohamed Gharieb (iD) ${ }^{1,2}$ \\ Hisham Samy Shalaby ${ }^{3}$ \\ Ihab Saad Othman ${ }^{4,5}$ \\ 'Faculty of Medicine, Ain Shams \\ University, Cairo, Egypt; ${ }^{2}$ Research and \\ Development, Eye World Hospital, Giza, \\ Egypt; ${ }^{3}$ Department of Ophthalmology, \\ Faculty of Medicine, Ain Shams \\ University, Cairo, Egypt; ${ }^{4}$ Eye World \\ Hospital, Giza, Egypt; ${ }^{5}$ Faculty of \\ Medicine, Cairo University, Giza, Egypt
}

\begin{abstract}
Purpose: This study aims to assess the intra-operator repeatability and correlation of the Pentacam HR (device 1) and Sirius (device 2) in measuring anterior segment parameters and to evaluate the agreement of their readings and therefore their interchangeability in a clinical setting.

Methods: This is a prospective non-randomized study was conducted on the right eyes of 102 subjects coming to Eye World Hospital, Giza, Egypt. With each machine, four scans were taken by a single examiner. Each device was used to measure keratometric indices, corneal thickness, anterior chamber depth, anterior chamber angle, corneal diameter and corneal optical aberrations.

Results: Both devices show high repeatability for corneal thickness, corneal diameter, anterior chamber depth and keratometric indices (except for maximum keratometry, where device 1 shows high repeatability and device 2 shows low repeatability). On the other hand, both devices show poor repeatability for anterior chamber angle, Q-values, root mean square, spherical, coma and trefoil aberrations. The readings of the two devices are strongly correlated as regards only keratometric indices, corneal thickness and anterior chamber depth. In addition, the readings of the devices are in good agreement as regards only keratometric indices (except maximum keratometry), corneal thickness, anterior chamber depth, anterior chamber angle, root mean square, spherical and trefoil aberrations.

Conclusion: Both devices showed variable intra-observer repeatability, with the device 1 showing slightly higher repeatability. Despite the similarity between some of the readings of the two devices, caution is advised before considering them interchangeable. We therefore do not recommend using them in alternation in refractive surgery.
\end{abstract}

Keywords: Scheimpflug, wavefront, corneal topography

\section{Introduction}

Precise assessment of the corneal power, corneal thickness and anterior chamber depth has become essential for anterior segment surgery. Since the dawn of corneal refractive surgery, several topography devices have been introduced. The surgeon must have a precise assessment of anterior segment measurements, in addition to excellent repeatability by each device for proper preoperative evaluation. ${ }^{1-4}$

The Pentacam (Oculus Inc., Wetzlar, Germany) was introduced in 2005 and uses a scanning slit with a Scheimpflug camera, giving images of the cornea, lens, and
Correspondence: Hisham Samy Shalaby Department of Ophthalmology, Faculty of Medicine, Ain Shams University, Abbassia Square, Cairo II59I, Egypt

Tel +20 1003641789

Email hisham_ophth@yahoo.com 
iris, therefore allowing corneal, anterior chamber and lens anatomy to be analyzed. The Pentacam machine uses a rotating Scheimpflug camera and a monochromatic slitlight source that rotate around the optical axes of the eye to generate a three-dimensional model of the anterior segment. Twenty-five images are captured within 2 seconds, with each slit image composed of 25,000 points including 500 true elevation points. ${ }^{5}$ The Sirius system (CSO Costruzione Strumenti Oftalmici, Florence, Italy) combines a monochromatic $360^{\circ}$ rotating Scheimpflug camera with a Placido disk-based corneal topographer, to better analyze the anterior corneal curvature. The system can measure 35,632 points from the anterior cornea and 30,000 points from the posterior corneal surface. A pachymetric map is therefore created using the data from both surfaces within 5 to 6 seconds of acquisition time. ${ }^{5,6}$

We aim through this study to compare the anterior chamber, topographic and corneal aberration parameters obtained with the Sirius to those obtained with the Pentacam and to assess the repeatability of the measurements of each device and to evaluate if they can be rendered clinically fungible.

\section{Materials and Methods}

A prospective non-randomized study was conducted in Eye World Hospital, Giza, Egypt, after approval by Ain Shams University Ethical Committee and in accordance with the 1975 Helsinki declaration, as revised in 1983. The study was conducted between April 2019 and August 2019. A written informed consent was taken from each participating subject, prior to recruitment. The right eyes of 102 subjects were enrolled from patients visiting the Eye World Hospital seeking refractive surgery. One eye per subject was enrolled to avoid inter-eye correlation bias. Since the variance between eyes is usually less than that between subjects, the overall variance of a sample of measurements combined from both eyes is likely to be an underestimate of the true variance resulting in an increased risk of rejecting the null hypothesis when it is true. ${ }^{7}$ We included individuals at least 18 years of age or older, with refractive errors ranging from -8.50 to +6.00 diopter sphere and up to 4.50 diopters cylinder. Exclusion criteria were any corneal pathology (including dry eye, which was identified using slit-lamp examination and advanced tear film analysis on the Sirius device), previous intraocular surgery, corneal scars, glaucoma, and history of wearing contact lenses within the last month before the study. The patients were subjected to complete ocular examination including best corrected visual acuity, refraction, slit-lamp biomicroscopy, and intraocular pressure measurement using a non-contact air puff tonometer.

All shots by both the Pentacam (device 1) and Sirius (device 2) were taken between 5 and 9 pm with the subject awake for at least 6 hours prior to measurement. ${ }^{8}$ With each machine, four reliable scans were taken by a singleexperienced examiner. For the Sirius device (CSO, Costruzione Strumenti Oftalmici, Florence, Italy, version 3.2.1.60) manual acquisition was used, while with the Pentacam (Oculus Inc., nd Wetzlar, Germany, version 1.21r.65) automated scans were acquired. Images with quality factor $<95 \%$ were excluded. Each device was used to collect the following data from each subject:

1. Flat anterior surface keratometry (K1)

2. Steep anterior surface keratometry (K2)

3. Flat posterior surface keratometry (K1-back)

4. Steep posterior surface keratometry (K2-back)

5. Maximum keratometry (K-max)

6. Central corneal thickness (CCT)

7. Thinnest location thickness (TLT)

8. Q-value of the anterior surface (Q-front)

9. Q-value of the posterior surface (Q-back)

10. Anterior chamber depth (ACD)

11. Anterior chamber angle (ACA)

12. Corneal diameter (CD)

13. Root mean square value of total corneal aberrations (RMS-T)

14. Root mean square of low order aberrations (RMSLOA)

15. Root mean square of high order aberrations (RMSHOA)

16. Spherical aberrations (SA)

17. Horizontal coma aberrations (Coma-0)

18. Vertical coma aberrations (Coma-90)

19. Oblique Trefoil aberrations (Trefoil-0)

20. Vertical Trefoil aberrations (Trefoil-30)

\section{Statistical Analysis}

Data were statistically analyzed using IBM SPSS Statistical Package for the Social Sciences version 22 (SPSS, Inc., Chicago, IL, USA) and graphs were formulated using MedCalc Statistical Software version 18.9.1 (MedCalc Software bvba, Ostend, Belgium). 
Sample size was calculated by $G^{*}$ Power v. 3.1.9.7 software. Power was set at 0.95 and $\alpha$ error 0.05. Sample size was 85 subjects. Data were tested for normality using Kolmogorov-Smirnov test with Lilliefors Significance Correction.

Age and gender were described as frequency while other data were described as mean \pm SD (range) with $95 \%$ confidence interval $(95 \% \mathrm{CI})$.

We assessed repeatability of examinations of each machine by within-subject standard deviation (SW) which was calculated as the square root of the within-subject mean square error, test-retest repeatability (TRT) which was calculated as $1.96 \sqrt{2}(\approx 2.77) * \mathrm{SW}$ and within-subject coefficient of variation $(\mathrm{COV}=100 \times \mathrm{SW} /$ overall mean $)$.

Correlation between devices was done using Pearson correlation coefficient and the intraclass correlation coefficient (ICC) which is a coefficient of reliability of data between different measurements; the closer the ICC is to 1 , the better the consistency of measurement is present. ICC ranges from low to high as follows: (ICC $<0.75$ : low reliability, $0.75 \leq \mathrm{ICC} \leq 0.90$ : moderate reliability and ICC $>0.9$ : high reliability). The BlandAltman Plots were used to determine the $95 \%$ limits of agreement (95\% LOA) between the measurements of the two devices. A P-value $<0.05$ was considered statistically significant.

\section{Results}

This study was performed on the right eyes of 102 patients presenting to Eye World Hospital, Giza, Egypt.

\section{Demographic Data}

Fifty-eight of the subjects (57.4\%) were females, while 43 $(42.6 \%)$ were males. The average age was $30.4( \pm 7.4 \mathrm{SD})$, with a range of 18 to 54 years old.

\section{Clinical Examination}

Subjective refraction of the 102 subjects revealed that the spherical error ranged from +6.5 to -8.5 dioptres (mean -2.1 dioptres $\pm 2.3 \mathrm{SD}$ ). The astigmatic error ranged from 0.0 to -4.5 dioptres (mean -1.2 dioptres $\pm 0.9 \mathrm{SD}$ ).

The uncorrected distance visual acuity (UDVA) ranged from 0.05 to 1.0 (20/400 to $20 / 20$ Snellen), mean 0.26 $\pm 0.18 \mathrm{SD}$, while the corrected distance visual acuity (CDVA) ranged from 0.2 to $2.0 \quad(20 / 100$ to $40 / 20$ Snellen), mean $0.9 \pm 0.3 \mathrm{SD}$.
Repeatability of Scans of Devices I and 2

Table 1 shows the mean, 95\% confidence interval, within subject standard deviation (SW), test-retest-repeatability (TRT) and coefficient of variation for parameters measured by the two devices. Both devices show high repeatability in the form of low variance (coefficient of variation $<1 \%$ ) for K1, K2, K1-back, K2-back, CCT, TLT, CD and ACD. Furthermore, both devices show poor repeatability in the form of high variance (coefficient of variation $>1 \%$ ) for ACA, Q-front, Q-back, SA, RMS-T, RMS-LOA, RMSHOA, Coma-0, Coma-90, Trefoil-0 and Trefoil-30. For K-max however, device 1 shows low variance and device 2 shows high variance. On comparing the repeatability of the two devices, the device 1 shows better repeatability for K1, K2, K-max, Q-front, ACD, CD, Trefoil-0 and Trefoil30. On the other hand, the device 2 shows better repeatability for the K1-back, K2-back, CCT, TLT, ACA, RMS-T, RMS-LOA, RMS-HOA, SA, Coma-0 and Coma-90.

\section{Correlation Between Device I and Device 2 Readings}

Table 2 shows the Pearson Correlation Coefficient and the Intraclass Correlation Coefficient (which takes into account the systematic error in comparing the readings of the two devices) to assess the correlation between readings taken by the device 1 and device 2 (where $r=1$ is total positive linear correlation, $r=0$ is no linear correlation, and $\mathrm{r}=-1$ is total negative linear correlation). The correlation between the two devices was variable regarding the various parameters: The readings of the two devices are strongly correlated as regards $\mathrm{K} 1, \mathrm{~K} 2$, K1-back, K2back, K-max, CCT, TLT and ACD. This correlation is relatively weaker for Q-front, Q-back, ACA, CD, RMST, RMS-LOA, RMS-HOA, SA, Trefoil-0 and Trefoil-30. Moreover, the two devices show anti-correlation for the Coma-0 and Coma-90 values. This is also shown in Figures 1 and 2.

\section{Agreement Between Device I and Device 2 Readings}

A Bland-Altman plot was used to illustrate the agreement between the measurements of the two machines, as shown in Table 3 and Figures 3 and 4. ${ }^{9}$ Clinical correlation shows that the readings of devices 1 and 2 are in good agreement as regards $\mathrm{K} 1, \mathrm{~K} 2$, K1-back, K2-back, CCT, TLT, ACD, ACA, RMS-T, RMS-LOA, RMS-HOA, SA, Trefoil-0 and Trefoil-30. However, the devices are in poor agreement as 


\begin{tabular}{|c|c|c|c|c|c|c|c|c|c|c|c|c|c|}
\hline 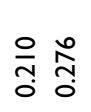 & 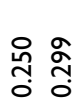 & 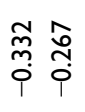 & 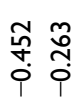 & $\begin{array}{l}\text { o̊ } \\
\text { gे } \\
0\end{array}$ & $\begin{array}{l}\text { م్ } \\
\text { م̂े }\end{array}$ & 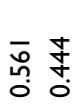 & $\begin{array}{ll}\hat{o} & 0 \\
0 \\
0 \\
0\end{array}$ & 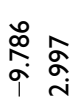 & $\begin{array}{l}\text { مू } \\
\hat{h} \\
0\end{array}$ & 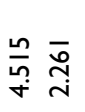 & $\underset{\substack{* \\
0}}{\stackrel{\forall}{0}}$ & 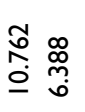 & 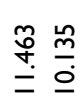 \\
\hline 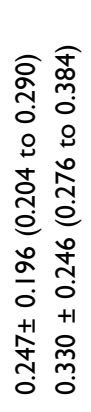 & 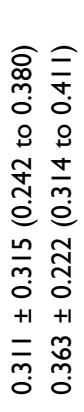 & 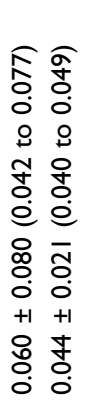 & 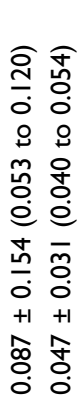 & 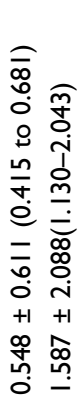 & 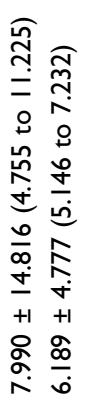 & 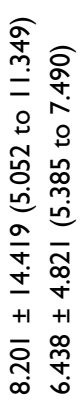 & 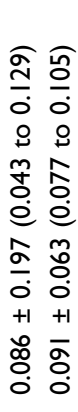 & 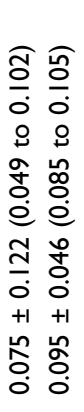 & 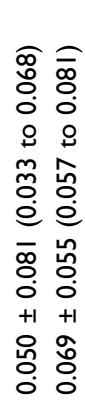 & 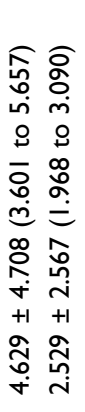 & 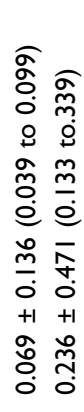 & 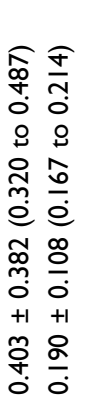 & 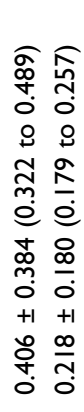 \\
\hline 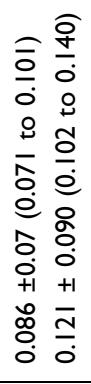 & 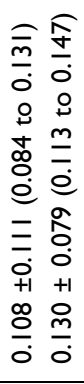 & 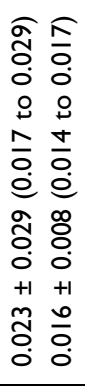 & 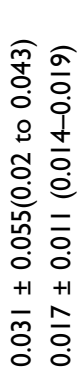 & 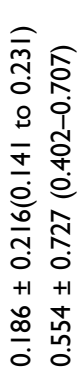 & 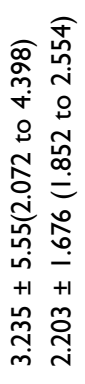 & 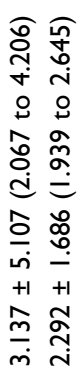 & 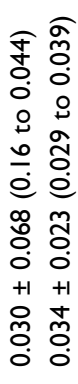 & 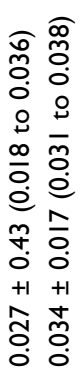 & 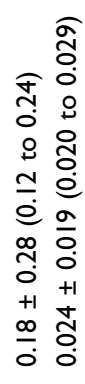 & 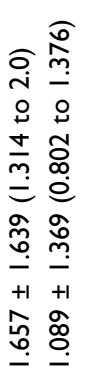 & 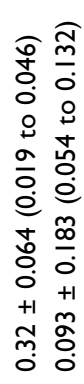 & 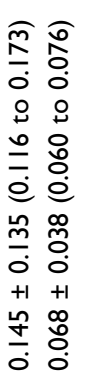 & 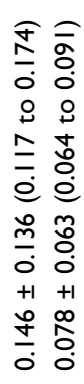 \\
\hline 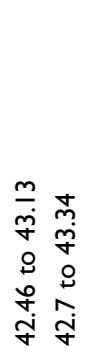 & 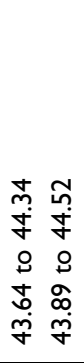 & 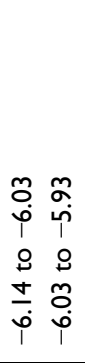 & 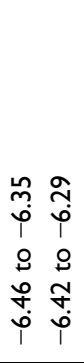 & 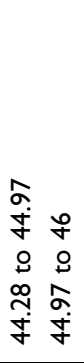 & 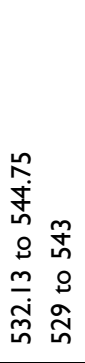 & 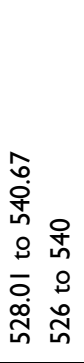 & 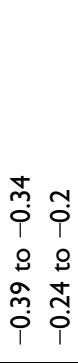 & 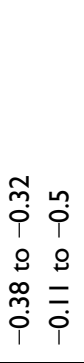 & 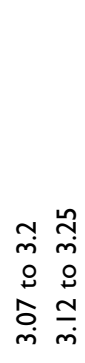 & 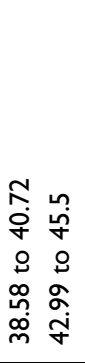 & 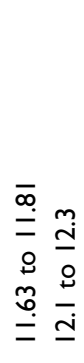 & 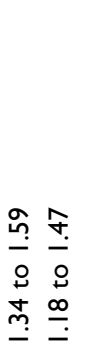 & 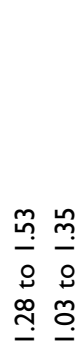 \\
\hline 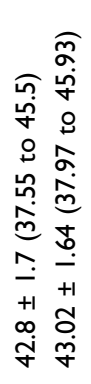 & 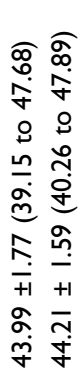 & 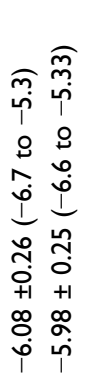 & 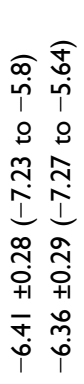 & 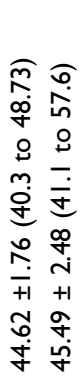 & 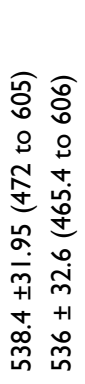 & 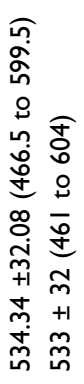 & 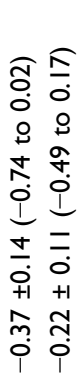 & 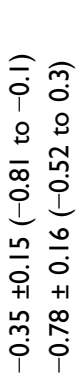 & 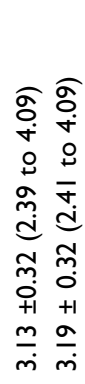 & 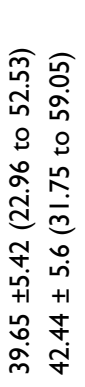 & 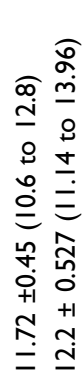 & 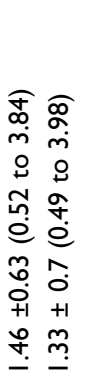 & 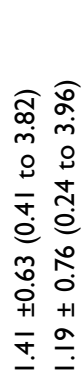 \\
\hline D̃ & $\overline{0}$ & $\bar{~}$ & 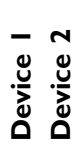 & 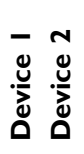 & 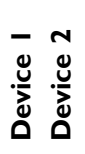 & ס̊ & סू & סू & 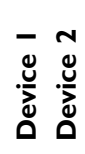 & סू & 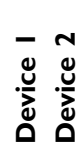 & 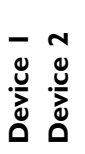 & 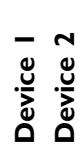 \\
\hline$\overline{\underline{x}}$ & $\underline{\underline{z}}$ & 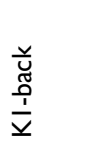 & 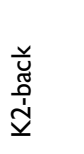 & 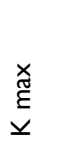 & U & $\vec{r}$ & ó & 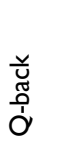 & U & 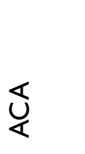 & u & $\stackrel{\curvearrowright}{\propto}$ & بَ \\
\hline
\end{tabular}




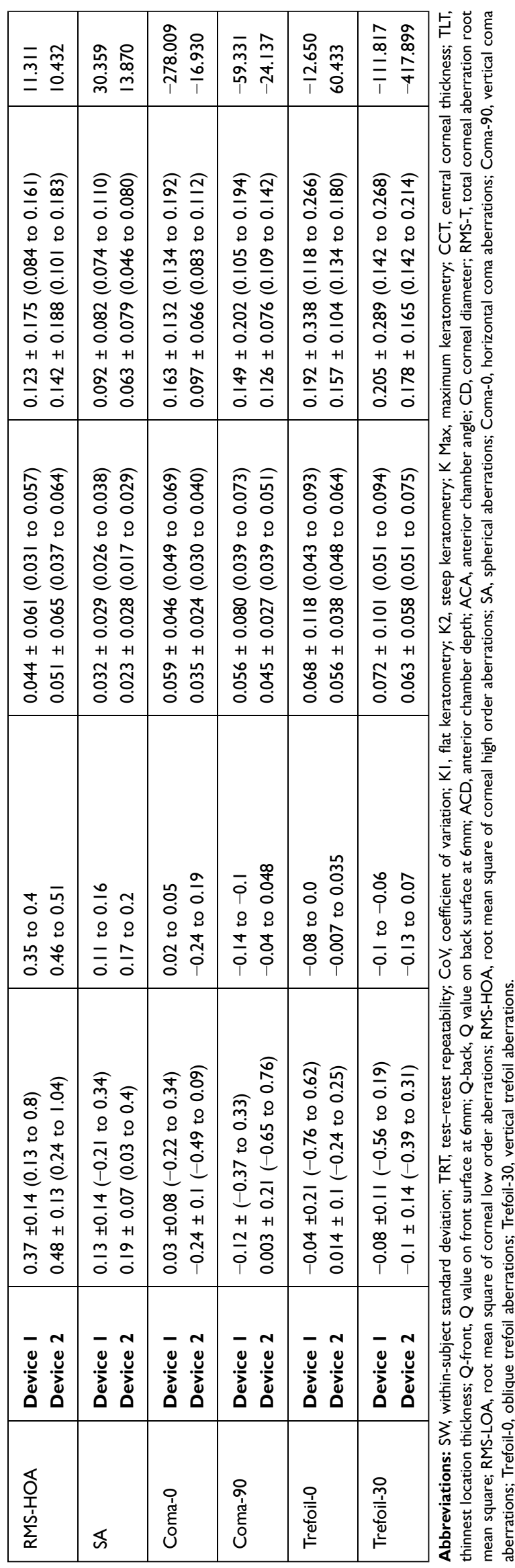

regards K-max, CD, Q-front, Q-back, Coma-0 and Coma-90.

\section{Discussion}

Refractive surgery is a rapidly growing field of ophthalmology that relies heavily on accurate measurement of anterior segment parameters. Corneal and anterior segment imaging has become crucial in clinical ophthalmology, with various applications, such as analysis of corneal curvature and pachymetry, detection of keratoconus, or study of the anterior segment before phakic intraocular lens implantation. ${ }^{10}$ Several devices are now available that permit recording such parameters. This study evaluates the reliability of the Pentacam and Sirius devices to produce repeatable results when measuring anterior segment characteristics, in addition to the agreement of the readings between the two devices to determine their interchangeability. It therefore raises the question whether the refractive surgeon has the choice of shifting between the two devices during clinical follow up of either anterior segment parameters or wavefront aberrations.

The Pentacam (device 1) showed good repeatability in the form of low variance (coefficient of variation $<1 \%$ ) for K1, K2, K1-back, K2-back, K-max, CCT, TLT, ACD and $\mathrm{CD}$, but poor repeatability in the form of high variance (coefficient of variation $>1 \%$ ) for Q-front, Q-back, ACA, RMS-T, RMS-LOA, RMS-HOA, SA, Coma-0, Coma-90, Trefoil-0 and Trefoil-30. This is in concurrence with the other studies. De la Parra-Colin et $\mathrm{al}^{11}$ showed that the Pentacam device gives repeatable results for steep K, flat $\mathrm{K}$, central corneal thickness, thinnest corneal thickness and anterior chamber depth. The same was revealed in a study by Kumar et al, ${ }^{5}$ who demonstrated that Pentacam has high repeatability for flat K, steep K, central corneal thickness, thinnest corneal thickness and anterior chamber depth. In addition, Crawford et $\mathrm{al}^{12}$ proved that Pentacam readings are repeatable for steep $\mathrm{K}$, flat $\mathrm{K}$ and central corneal thickness. McAlinden et $\mathrm{al}^{13}$ observed that measurements taken with the Pentacam HR are repeatable and reproducible, but warned that corneal axes, pupil center pachymetry, front meridional and axial maps, refractive power maps, and equivalent $\mathrm{K}$ readings should be interpreted with caution Furthermore, Aramberri et $\mathrm{al}^{14}$ concluded that repeatability and reproducibility of Pentacam HR readings were good for all parameters including corneal wavefront aberrations. Several studies have evaluated the Pentacam's repeatability on central and peripheral 
Table 2 Pearson Correlation Coefficient and Intraclass Correlation Coefficient for Readings Taken by Devices I and 2

\begin{tabular}{|c|c|c|c|c|}
\hline & $\begin{array}{l}\text { Pearson Correlation } \\
\text { Coefficient } r\end{array}$ & $\begin{array}{l}\text { Pearson Correlation } \\
\text { Coefficient P-value }\end{array}$ & $\begin{array}{l}\text { Intraclass Correlation } \\
\text { Coefficient } r\end{array}$ & $\begin{array}{l}\text { Intraclass Correlation } \\
\text { Coefficient P-value }\end{array}$ \\
\hline $\mathrm{KI}$ & 0.9939 & $<0.0001$ & 0.996 & $<0.001$ \\
\hline $\mathrm{K} 2$ & 0.9922 & $<0.0001$ & 0.996 & $<0.001$ \\
\hline KI back & 0.9809 & $<0.0001$ & 0.946 & $<0.001$ \\
\hline K2 back & 0.9778 & $<0.0001$ & 0.980 & $<0.001$ \\
\hline $\mathrm{K} \max$ & 0.815 & $<0.0001$ & 0.836 & $<0.001$ \\
\hline CCT & 0.9827 & $<0.0001$ & 0.988 & $<0.001$ \\
\hline TLT & 0.9870 & $<0.0001$ & 0.992 & $<0.001$ \\
\hline Q-front & 0.6582 & $<0.0001$ & 0.508 & $<0.001$ \\
\hline Q-back & 0.5175 & $<0.0001$ & 0.328 & $<0.001$ \\
\hline$A C D$ & 0.9896 & $<0.0001$ & 0.989 & $<0.001$ \\
\hline $\mathrm{ACA}$ & 0.6923 & $<0.0001$ & 0.691 & $<0.001$ \\
\hline$C D$ & 0.5348 & $<0.0001$ & 0.442 & $<0.001$ \\
\hline RMS-T & 0.7017 & $<0.0001$ & 0.812 & $<0.001$ \\
\hline RMS-LOA & 0.6930 & $<0.0001$ & 0.787 & $<0.001$ \\
\hline RMS-HOA & 0.6158 & $<0.0001$ & 0.626 & $<0.001$ \\
\hline SA & 0.4173 & $<0.0001$ & 0.477 & $<0.001$ \\
\hline Coma-0 & -0.1144 & $\mathrm{P}=0.2830$ & -0.051 & $P=0.845$ \\
\hline Coma-90 & -0.06149 & $P=0.5648$ & -0.081 & $P=0.679$ \\
\hline Trefoil-0 & 0.05068 & $P=0.6352$ & 0.073 & $\mathrm{P}=0.356$ \\
\hline Trefoil-30 & 0.6927 & $<0.0001$ & 0.786 & $<0.001$ \\
\hline
\end{tabular}

Abbreviations: K1, flat keratometry; K2, steep keratometry; K Max, maximum keratometry; CCT, central corneal thickness; TLT, thinnest location thickness; Q-front, $Q$ value on front surface at $6 \mathrm{~mm}$; $Q$-back, $Q$ value on back surface at $6 \mathrm{~mm} ; A C D$, anterior chamber depth; $A C A$, anterior chamber angle; $C D$, corneal diameter; $R M S-T$, total corneal aberration root mean square; RMS-LOA, root mean square of corneal low order aberrations; RMS-HOA, root mean square of corneal high order aberrations; SA, spherical aberrations; Coma-0, horizontal coma aberrations; Coma-90, vertical coma aberrations; Trefoil-0, oblique trefoil aberrations; Trefoil-30, vertical trefoil aberrations.

corneal thicknesses ${ }^{15,16}$ and ACD. ${ }^{17}$ Our repeatability results for the Pentacam agree with these previous studies.

On the other hand, the Sirius (device 2) showed good repeatability in the form of low variance (coefficient of variation $<1 \%$ ) for $\mathrm{K} 1, \mathrm{~K} 2$, K1-back, K2-back, CCT, TLT, $\mathrm{ACD}$, and $\mathrm{CD}$, but poor repeatability (coefficient of variation $>1 \%$ ) for K-max, ACA, Q-front, Q-back, RMS-T, RMSLOA, RMS-HOA, SA, Coma-0, Coma-90, Trefoil-0 and Trefoil-30. Again, this was in accord with the results of De la Parra-Colin et al, ${ }^{11}$ who found the Sirius readings repeatable (coefficient of variation $<1 \%$ ) for steep $\mathrm{K}$, flat $\mathrm{K}$, central corneal thickness, thinnest corneal thickness and anterior depth. The same five parameters were again found repeatable (coefficient of variation $<1 \%$ ) when measured by the Sirius device in the study by Kumar et al. ${ }^{5}$ Likewise, Duman et al described good repeatability of readings by the Sirius device on measuring $\mathrm{K} 1, \mathrm{~K} 2, \mathrm{CCT}$ and $\mathrm{ACD}$ in cataractous patients, ${ }^{18}$ while Gokcinar et al reported highly repeatable Sirius readings for $\mathrm{CCT}^{19}{ }^{19}$ However, the study by Nasser et $\mathrm{al}^{20}$ concluded that minimal corneal thickness and posterior corneal radii at $3 \mathrm{~mm}$ and $7 \mathrm{~mm}$ have poor repeatability (coefficient of variation $>1 \%$ ), while anterior corneal radii and anterior chamber depth have good repeatability (coefficient of variation $<1 \%$ ). The lower repeatability noted in the latter study may be attributed to the smaller sample size of 45 eyes used by Nasser et al compared to our larger sample size of 102 eyes.

Comparing the two devices, we found the device 1 readings to be more repeatable than device 2 readings for K1, K2, K-max, Q-front, ACD, CD, Trefoil-0 and Trefoil30. On the other hand, device 2 shows better repeatability for the K1-back, K2-back, CCT, TLT, ACA, RMS-T, RMS-LOA, RMS-HOA, SA, Coma-0 and Coma-90.

Furthermore, the readings of the two devices were found to be strongly correlated as regards K1, K2, K1-back, K2-back, $\mathrm{K}-\mathrm{max}, \mathrm{CCT}$, TLT and ACD. This correlation is weaker for Q-front, Q-back, ACA, CD, RMS-T, RMS-LOA, RMS-HOA, SA, Trefoil-0 and Trefoil-30. Moreover, the two devices show anti-correlation for the Coma- 0 and Coma- 90 values. The study by De la Parra-Colin et $\mathrm{al}^{11}$ showed a similar strong correlation between the two devices as regards steep K, flat K, central corneal thickness and thinnest corneal thickness.

Evaluation of the agreement and interchangeability between the two devices shows that the readings of devices 

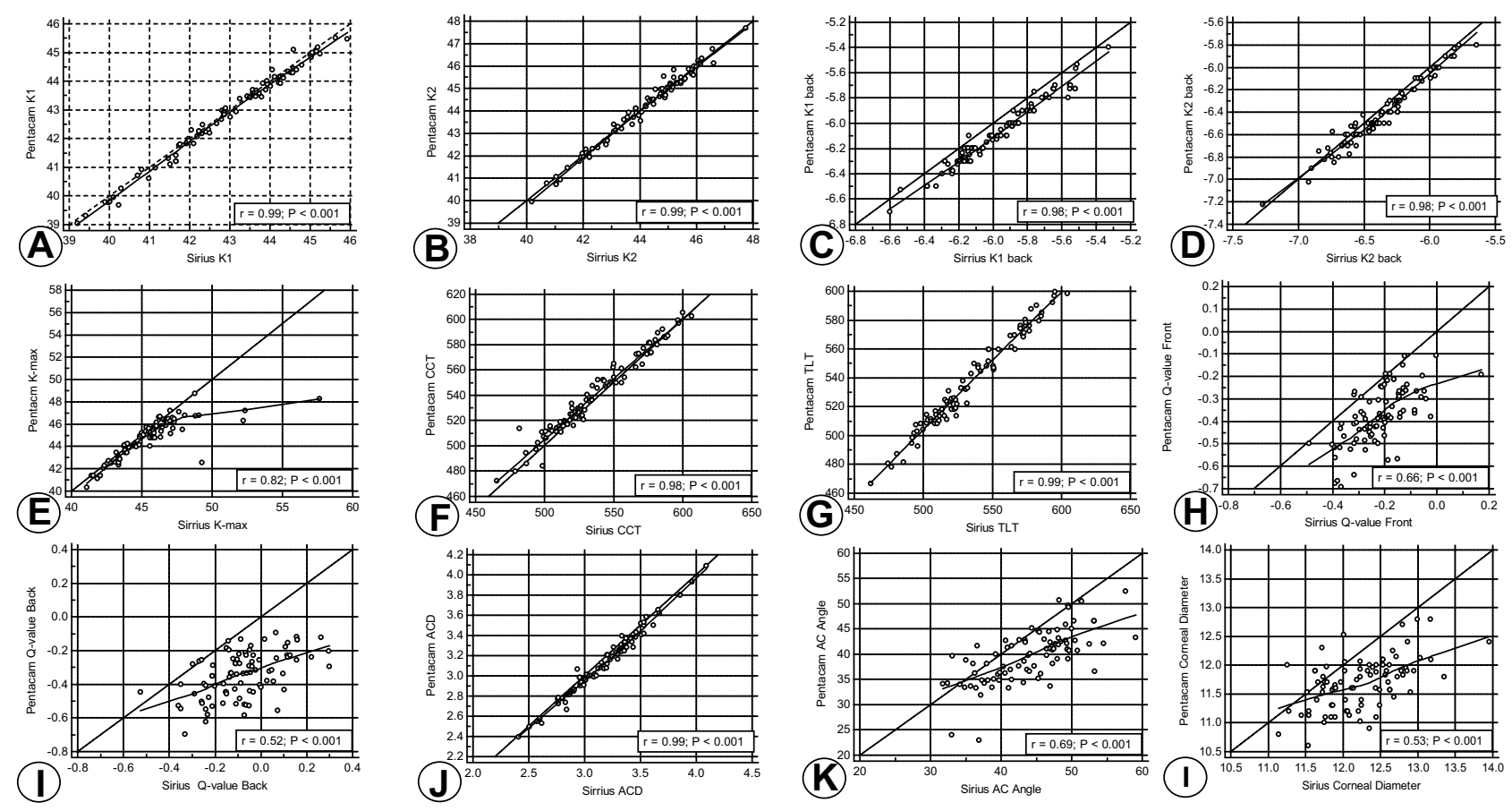

Figure I Pearson correlation between device I and device 2 for: (A) KI, (B) K2, (C) KI-back, (D) K2-back, (E) K-max, (F) CCT, (G) TLT, (H) Q-front, (I) Q-back, (J) $A C D,(\mathbf{K})$ ACA and (L) CD.
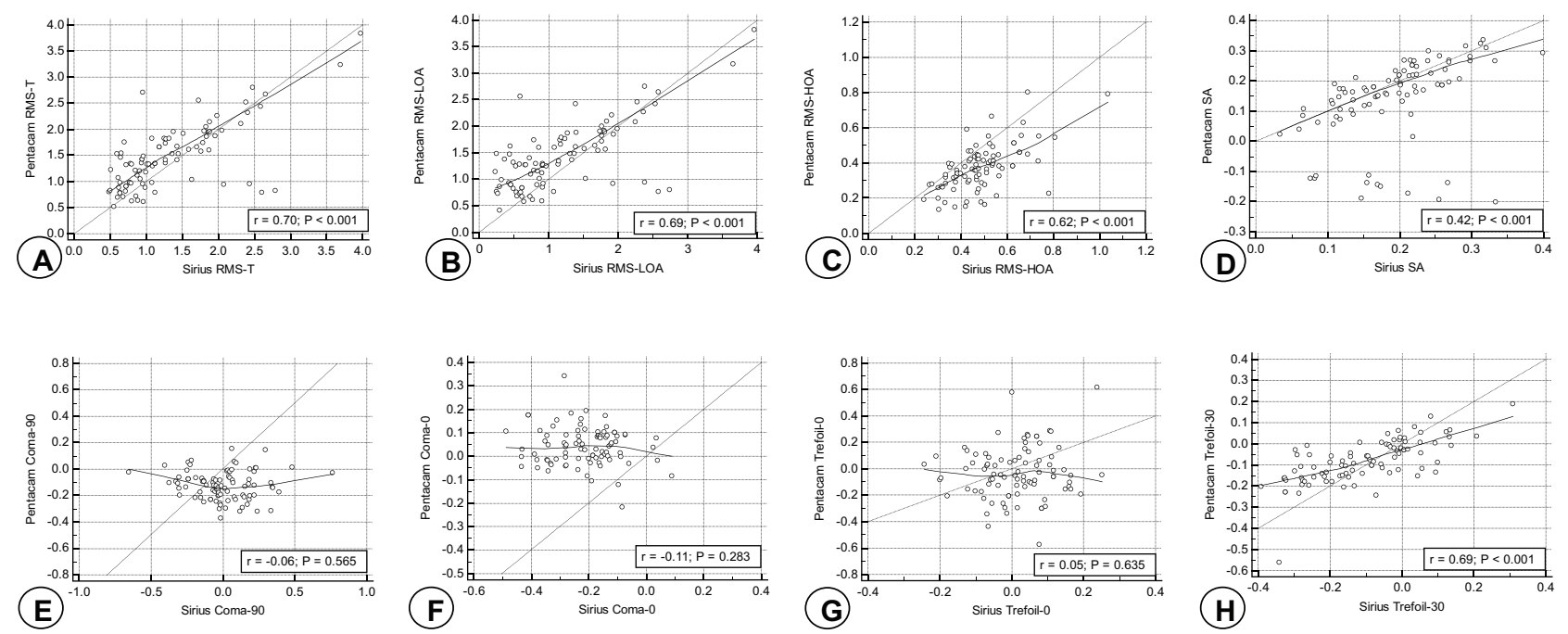

Figure 2 Pearson correlation between device I and device 2 for: (A) RMS-T, (B) RMS-LOA, (C) RMS-HOA, (D) SA, (E) Coma-90, (F) Coma-0, (G) Trefoil-30 and (H) Trefoil-0.

1 and 2 are in good agreement as regards $\mathrm{K} 1, \mathrm{~K} 2, \mathrm{~K} 1$-back, K2-back, CCT, TLT, ACD, ACA, RMS-T, RMS-LOA, RMS-HOA, SA, Trefoil-0 and Trefoil-30. However, the devices are in poor agreement as regards $\mathrm{K}-\mathrm{max}, \mathrm{CD}$, Q-front, Q-back, Coma-0 and Coma-90. In contrast to our results, De la Parra-Colin et $\mathrm{al}^{11}$ found that Sirius and Pentacam should not be considered exchangeable for steep
K, CCT and TLT. In addition, Kumar et $\mathrm{al}^{5}$ found that differences between Sirius and Pentacam for steep K, flat $\mathrm{K}, \mathrm{CCT}$, TLT and ACD were statistically significant $(\mathrm{P}<$ 0.05), with Sirius yielding higher measurements for steep K, flat $\mathrm{K}$ and ACD and Pentacam for TLT and CCT. This discrepancy in results from our findings could again be attributed to our larger sample size. Other studies also stress 
that Pentacam and Sirius should not be used interchangeably. $^{20-22}$

To our knowledge, there are no previous studies that assess the repeatability and interchangeability of the two Scheimpflug camera devices as regards wavefront and corneal optical aberrations analysis. Higher-order aberrations are clinically relevant as some dysphotopsias including night halos and glare have been associated with high amounts of higher-order aberrations. ${ }^{23}$ Therefore, this is a novel study which assesses their intra-observer repeatability and compares the agreement between the readings of the two machines. While other studies ${ }^{5,11,20-22}$ have compared their agreement as regards anterior segment parameters only, ours probes deeper to assess this agreement for wavefront aberrations. It answers the question whether the two machines could be used in clinical practice in alternation. Considering the two imaging systems interchangeable would lead to misinterpretation of clinical data that could alter decisions in refractive surgery.

However, we are aware that our findings may be affected by the wide range of age group. This shortcoming may be addressed in future studies. Another limitation is the fact that while refractive surgery is performed in the supine position, imaging by Scheimpflug devices is possible in the erect position only. Body posture has been shown to influence CCT measurements. ${ }^{24}$ Ultrasound probe pachymetry offers only point measurements and uses a contact technique (possibly altering corneal shape); Moreover, proper probe alignment by the examiner is critical for consistent readings. On the contrary, the portable OCT overcomes such shortcomings. Its portability in the supine position, plus the ability to record high-resolution scans for monitoring changes, and the ability for rapid and non-contact acquisition, make portable OCT imaging a valuable tool. ${ }^{25}$

Table 3 Mean Readings of Device I and Device 2, Difference and Mean Difference Between Device I and Device 2 and Limits of Agreement

\begin{tabular}{|c|c|c|c|c|}
\hline & Mean Device I & Mean Device 2 & Mean Difference & Limit of Agreement \\
\hline $\mathrm{KI}$ & 42.8 & 43.02 & -0.11 & 0.23 to -0.45 \\
\hline $\mathrm{K} 2$ & 43.99 & 44.21 & -0.03 & 0.37 to -0.42 \\
\hline $\mathrm{KI}$-back & -6.08 & -5.98 & -0.11 & -0.01 to 0.20 \\
\hline K2-back & -6.41 & -6.36 & -0.05 & 0.07 to -0.18 \\
\hline K-max & 44.62 & 45.49 & -0.8 & 2.1 to -3.7 \\
\hline СCT & 538.4 & 536 & 3.6 & 15.5 to -8.2 \\
\hline TLT & 534.34 & 533 & 2.6 & 12.8 to -7.7 \\
\hline Q-front & -0.37 & -0.22 & -0.16 & 0.03 to -0.35 \\
\hline Q-back & -0.35 & -0.78 & -0.26 & 0.02 to -0.55 \\
\hline$A C D$ & 3.13 & 3.19 & -0.05 & 0.04 to -0.14 \\
\hline ACA & 39.65 & 42.44 & -4.4 & 4.3 to -13.1 \\
\hline$C D$ & 11.72 & 12.2 & -0.54 & 0.39 to -1.46 \\
\hline RMS-T & 1.46 & 1.33 & 0.14 & 1.16 to -0.87 \\
\hline RMS-LOA & $\mathrm{I} .4 \mathrm{I}$ & 1.19 & 0.22 & 1.32 to -0.87 \\
\hline RMS-HOA & 0.37 & 0.48 & -0.11 & 0.11 to -0.33 \\
\hline SA & 0.13 & 0.19 & -0.05 & 0.19 to -0.29 \\
\hline Coma-0 & 0.03 & -0.24 & 0.26 & 0.54 to -0.03 \\
\hline Coma-90 & -0.12 & 0.003 & -0.13 & 0.35 to -0.61 \\
\hline Trefoil-0 & -0.04 & 0.014 & -0.05 & 0.39 to -0.50 \\
\hline Trefoil-30 & -0.08 & -0.1 & 0.02 & 0.22 to -0.19 \\
\hline
\end{tabular}

Abbreviations: SW, within-subject standard deviation; TRT, test-retest repeatability; CoV, coefficient of variation; KI, flat keratometry; K2, steep Keratometry; K Max, maximum keratometry; CCT, central corneal thickness; TLT, thinnest location thickness; Q-front, Q value on front surface at $6 \mathrm{~mm}$; Q-back, Q value on back surface at $6 \mathrm{~mm}$; $A C D$, anterior chamber depth; ACA, anterior chamber angle; CD, corneal diameter; RMS-T, total corneal aberration root mean square; RMS-LOA, root mean square of corneal low order aberrations; RMS-HOA, root mean square of corneal high order aberrations; SA, spherical aberrations; Coma-0, horizontal coma aberrations; Coma-90, vertical coma aberrations; Trefoil-0, oblique trefoil aberrations; Trefoil-30, vertical trefoil aberrations. 

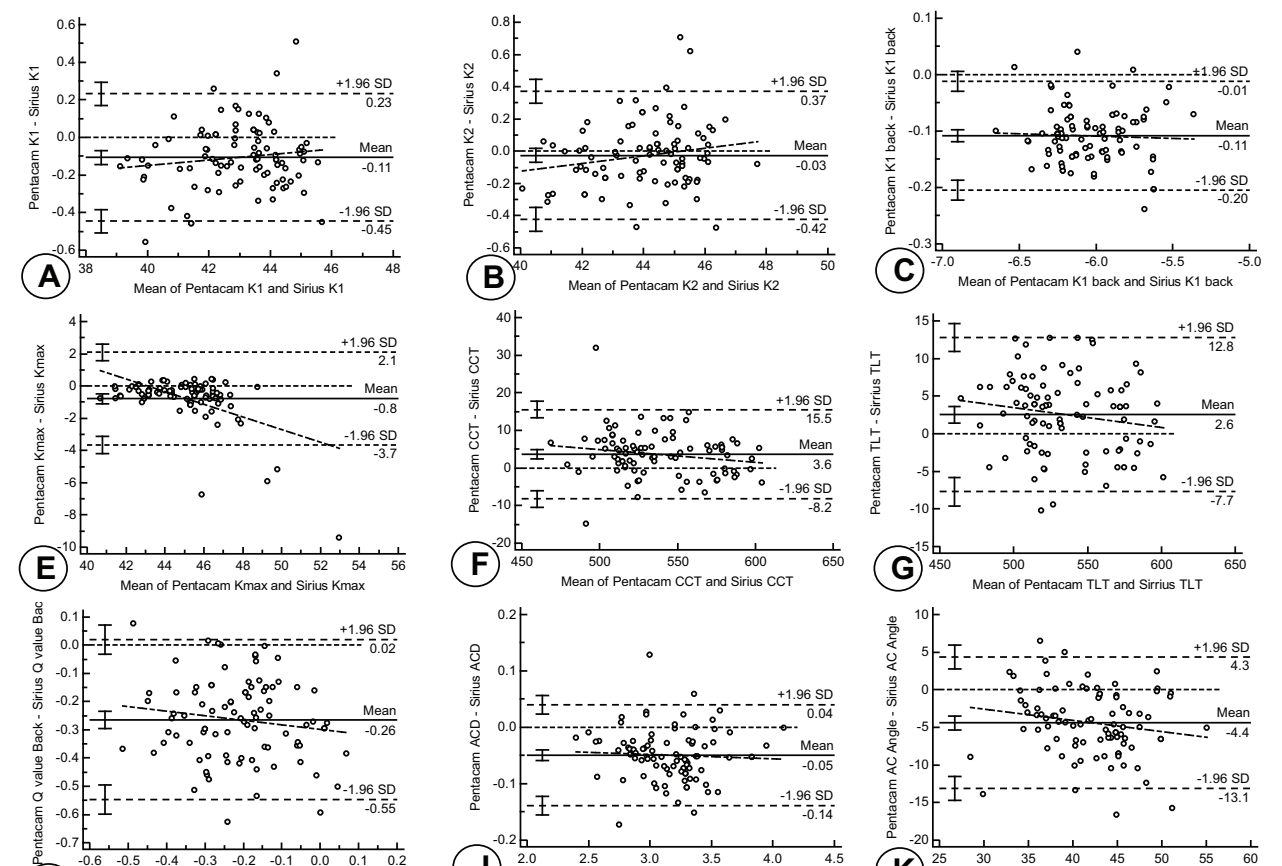

(I) Mean of Pentacam Q value Back and Sirius Q value Back
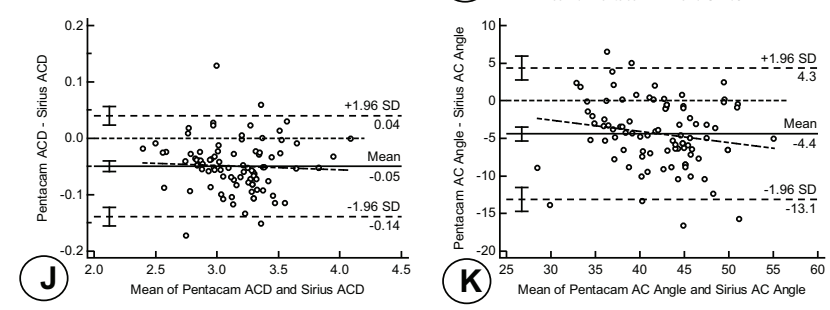
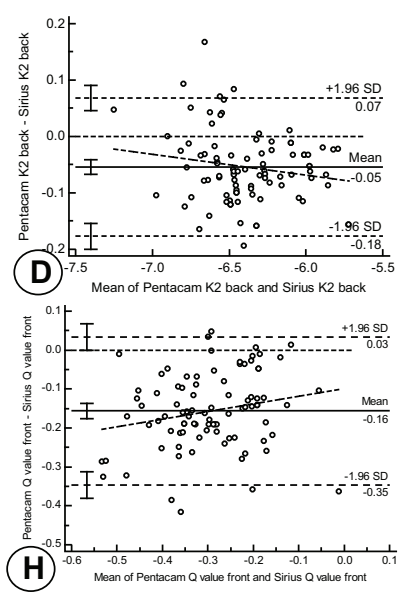

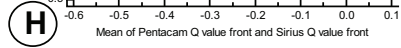

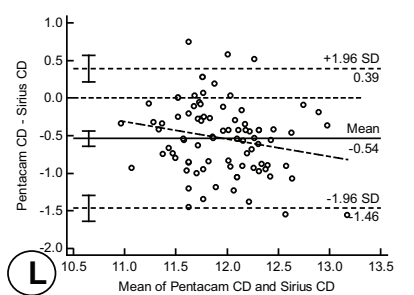

Figure 3 Bland-Altman plot showing agreement between device I and device 2 as regards KI (A), K2 (B), KI-back (C), K2-back (D), K-max (E), CCT (F) TLT (G), Q-front $(\mathbf{H})$, Q-back (I), ACD (J), ACA (K) and CD (L). The X-axis represents the mean of the readings recorded by both devices (device I + device $2 / 2)$, while the $Y$-axis represents the difference between the mean device I and device 2 readings (device I - device 2 ). The mean difference is calculated and is represented by the solid line. The closer the line is to 0 , the greater the agreement between the two devices. The mean difference lies between the upper and lower limits of agreement, representing +1.96 SD and - I.96 SD, respectively.

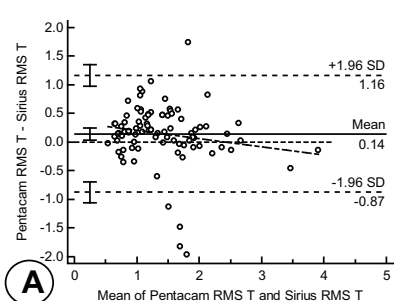

(A)

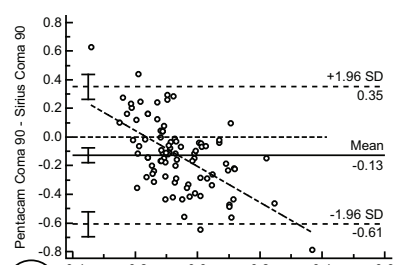

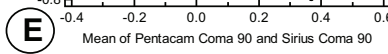

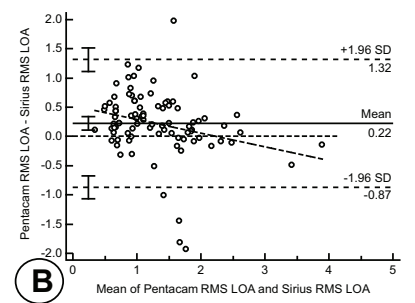

(F)

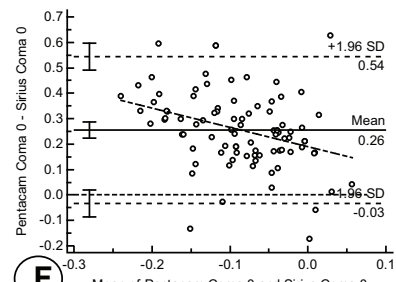

(B) Mean of Pentacam RMS LOA and Sirius RMS LOA

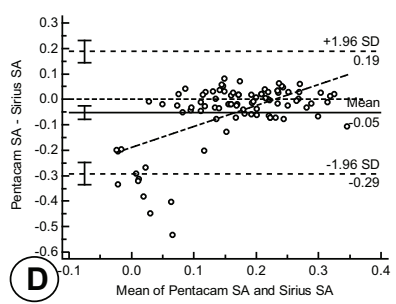

(C)

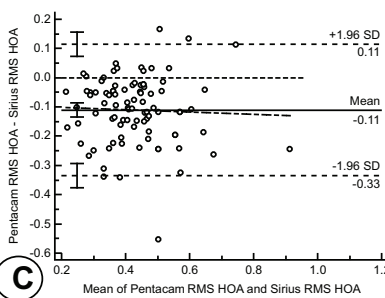

(D)
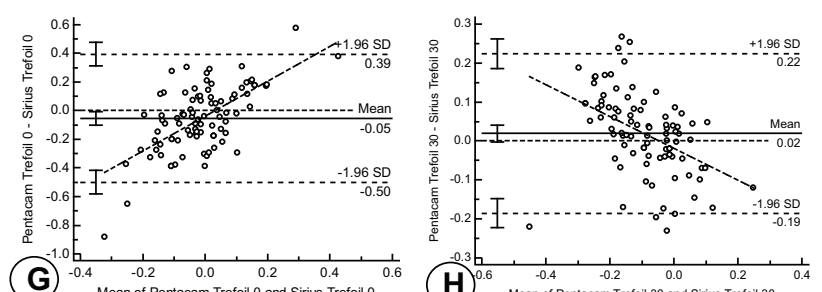

Figure 4 Bland-Altman plot showing agreement between device I and device 2 as regards RMS-T (A), RMS-LOA (B), RMS-HOA (C), SA (D), Coma-90 (E), Coma-0 (F), Trefoil-90 (G) and Trefoil-0 $(\mathbf{H})$. The X-axis represents the mean of the readings recorded by both devices (device I + device $2 / 2$ ), while the $Y$-axis represents the difference between the mean device I and device 2 readings (device I - device 2). The mean difference is calculated and is represented by the solid line. The closer the line is to 0 , the greater the agreement between the two devices. The mean difference lies between the upper and lower limits of agreement, representing + I.96 SD and -I.96 SD, respectively. 


\section{Disclosure}

The research was not funded. The authors indicate no relationships, conditions, or circumstances that present a potential financial conflict of interest and no financial disclosures. The authors report no conflicts of interest for this work.

\section{References}

1. Konstantopoulos A, Hossain P, Anderson DF. Recent advances in ophthalmic anterior segment imaging: a new era for ophthalmic diagnosis? Br J Ophthalmol. 2007;91(4):551-557.

2. Rao HL, Kumar AU, Kumar A, et al. Evaluation of central corneal thickness measurement with RTVue spectral domain optical coherence tomography in normal subjects. Cornea. 2011;30(2):121-126. doi:10.1097/ICO.0b013e3181e16c65

3. Dutta D, Rao HL, Addepalli UK, Vaddavalli PK. Corneal thickness in keratoconus: comparing optical, ultrasound, and optical coherence tomography pachymetry. Ophthalmology. 2013;120(3):457-463. doi:10.1016/j.ophtha.2012.08.036

4. Reznicek L, Muth D, Kampik A, Neubauer AS, Hirneiss C. Evaluation of a novel scheimpflug-based non-contact tonometer in healthy subjects and patients with ocular hypertension and glaucoma. Br J Ophthalmol. 2013;97(11):1410-1414. doi:10.1136/bjophthalmol-2013-303400

5. Kumar M, Shetty R, Jayadev C, Rao HL, Dutta D. Repeatability and agreement of five imaging systems for measuring anterior segment parameters in healthy eyes. Indian J Ophthalmol. 2017;65(4):288. doi:10.4103/ijo.IJO_729_16

6. Milla M, Piñero DP, Amparo F, Alió JL. Pachymetric measurements with a new scheimpflug photography-based system. J Cataract Refract Surg. 2011;37(2):310-316. doi:10.1016/j.jcrs.2010.08.038

7. Glynn RJ, Rosner B. Accounting for the correlation between fellow eyes in regression analysis. Arch Ophthalmol. 1992;110(3):381-387. doi:10.1001/archopht.1992.01080150079033

8. Feng Y, Varikooty J, Simpson TL. Diurnal variation of corneal and corneal epithelial thickness measured using optical coherence tomography. Cornea. 2001;20(5):480-483. doi:10.1097/00003226200107000-00008

9. Bland JM, Altman DG. Agreement between methods of measurement with multiple observations per individual. J Biopharm Stat. 2007;17 (4):571-582. doi:10.1080/10543400701329422

10. Piñero DP. Technologies for anatomical and geometric characterization of the corneal structure and anterior segment: a review. Semin Ophthalmol. 2015;30(3):161-170. doi:10.3109/ 08820538.2013.835844

11. De la Parra-colín P, Garza-León M, Barrientos-Gutierrez T. Repeatability and comparability of anterior segment biometry obtained by the sirius and the pentacam analyzers. Int Ophthalmol. 2014;34(1):27-33. doi:10.1007/s10792-013-9780-0

12. Crawford AZ, Patel DV, McGhee CN. Comparison and repeatability of keratometric and corneal power measurements obtained by Orbscan II, Pentacam, and Galilei corneal tomography systems. Am J Ophthalmol. 2013;156(1):53-60. doi:10.1016/j.ajo.2013.01.029
13. McAlinden C, Khadka J, Pesudovs K. A comprehensive evaluation of the precision (repeatability and reproducibility) of the Oculus Pentacam HR. Invest Ophthalmol Vis Sci. 2011;52(10):7731-7737. doi:10.1167/iovs.10-7093

14. Aramberri J, Araiz L, Garcia A, et al. Dual versus single scheimpflug camera for anterior segment analysis: precision and agreement. J Cataract Refract Surg. 2012;38(11):1934-1949. doi:10.1016/j. jcrs.2012.06.049

15. O'Donnell C, Maldonado-Codina C. Agreement and repeatability of central thickness measurement in normal corneas using ultrasound pachymetry and the OCULUS Pentacam. Cornea. 2005;24 (8):920-924. doi:10.1097/01.ico.0000157422.01146.e9

16. Lackner B, Schmidinger G, Pieh S, Funovics MA, Skorpik C. Repeatability and reproducibility of central corneal thickness measurement with Pentacam, Orbscan, and ultrasound. Optom Vis Sci. 2005;82(10):892-899. doi:10.1097/01.opx.0000180817.46312.0a

17. Lackner B, Schmidinger G, Skorpik C. Validity and repeatability of anterior chamber depth measurements with Pentacam and Orbscan. Optom Vis Sci. 2005;82(9):858-861. doi:10.1097/01. opx.0000177804.53192.15

18. Duman R, Çetinkaya E, Duman R, Dogan M, Sabaner MC. Comparison of anterior segment measurements using sirius topographer $^{\circledR}$ and Nidek Axial Length-Scan ${ }^{\circledR}$ with assessing repeatability in patients with cataracts. Indian J Ophthalmol. 2018;66 (3):402-406.

19. Gokcinar NB, Yumusak E, Ornek N, Yorubulut S, Onaran Z. Agreement and repeatability of central corneal thickness measurements by four different optical devices and an ultrasound pachymeter. Int Ophthalmol. 2019;39:1589-1598. doi:10.1007/s10792-018-0983-2

20. Nasser CK, Singer R, Barkana Y, Zadok D, Avni I, Goldich Y. Repeatability of the sirius imaging system and agreement with the Pentacam HR. J Refract Sur. 2012;28(7):493-497. doi:10.3928/ 1081597X-20120619-01

21. Savini G, Carbonelli M, Sbreglia A, Barboni P, Deluigi G, Hoffer KJ. Comparison of anterior segment measurements by 3 scheimpflug tomographers and 1 Placido corneal topographer. J Cataract Refract Surg. 2011;37(9):1679-1685. doi:10.1016/j.jcrs.2011.03.055

22. Anayol MA, Güler E, Yagc R, et al. Comparison of central corneal thickness, thinnest corneal thickness, anterior chamber depth, and simulated keratometry using galilei, Pentacam, and Sirius devices. Cornea. 2014;33(6):582-586. doi:10.1097/ICO.0000000000000119

23. Lee H, Park SY, Kang DSY, et al. Photorefractive keratectomy combined with corneal wavefront-guided and hyperaspheric ablation profiles to correct myopia. $J$ Cataract Refract Surg. 2016;42:890-898. doi:10.1016/j.jcrs.2016.03.033

24. Maslin JS, Teng CC, Chadha N, Liu J. Effect of supine body position on central corneal thickness. Clin Exp Ophthalmol. 2016;44 (8):678-683. doi:10.1111/ceo.12742

25. Napoli PE, Nioi M, Gabiati L, et al. Repeatability and reproducibility of post-mortem central corneal thickness measurements using a portable optical coherence tomography system in humans: a prospective multicenter study. Sci Rep. 2020;10:14508. doi:10.1038/s41598-020-71546-1
Clinical Ophthalmology

\section{Publish your work in this journal}

Clinical Ophthalmology is an international, peer-reviewed journal covering all subspecialties within ophthalmology. Key topics include: Optometry; Visual science; Pharmacology and drug therapy in eye diseases; Basic Sciences; Primary and Secondary eye care; Patient Safety and Quality of Care Improvements. This journal is indexed on PubMed
Central and CAS, and is the official journal of The Society of Clinical Ophthalmology (SCO). The manuscript management system is completely online and includes a very quick and fair peer-review system, which is all easy to use. Visit http://www.dovepress.com/ testimonials.php to read real quotes from published authors. 